\title{
Inhaltsverzeichnis
}

Vorwort

XIII

Keynote

Ed H. Chi

Model-Driven Research in Human-Computer Interaction 3

Beiträge

\section{Neue Interaktionstechnologien}

Sophie Stellmach, Thomas Brücher, Ronny Franke, Raimund Dachselt

Digitale Stift- und Papierinteraktion in Virtuellen Umgebungen

Thomas Bader, Astrid Heck

Lift-and-Drop: Lückenlose Interaktion über Displaygrenzen hinweg. .17

Marcus Specht, Andrea Söter, Jens Gerken, Hans-Christian Jetter, Lorenz Bohrer,

Harald Reiterer

Dynamic Force Fields zur Präzisionserhöhung von Zeigegeräten .27

\section{Kooperation in der Unternehmenspraxis}

Claudia Müller, Volkmar Pipek, Christian Reuter

Globale Infrastruktur - lokales Arbeiten: Praxis IT-gestützter Wartung bei einem

Energieversorger

Philipp Nussbaumer, Gerhard Schwabe

Gemeinsam statt einsam: Kooperative Bankberatung

Sascha Bingenheimer, Tom Gross

Towards Flexible Support for Dynamic and Interwoven Small Companies .57

\section{Schnittstellengestaltung im Web}

Ekaterina Karavaeva, Susanne Maaß

Benutzerorientierte Revision einer Informationsarchitektur .63

Dirk Lewandowski, Eva Nesbach, Nina Mikley

Lokale Suchmaschinen: Evaluierung und Gestaltungsempfehlungen.....

Tim Hussein, Werner Gaulke, Anabell Hartmann, Jürgen Ziegler

Wahrnehmung, Nutzung und Akzeptanz von systemgenerierten Produktempfehlungen .83 


\section{Sicherheit \& Gesundheit}

Tobias Schwarz, Flavius Kehr, Holger Oortmann, Harald Reiterer

Die Leitwarte von heute verstehen - die Leitwarte von morgen gestalten! .93

Britta Hennecken, Oliver Witt, Jessica Schwarz

Makro-Ergonomische Unterstützung in Operationszentralen der Deutschen Marine

Steven Birr, Volker Dicken, Bernhard Preim

Webbasierte Planungsunterstützung von Lungenoperationen

\section{Menschen interagieren mit Menschen}

Mirko Fetter, Julian Seifert, Tom Gross

Vorhersagbarkeit von Selektiver Verfügbarkeit im Instant Messaging

Stefanie Pötzsch

Einfluss wahrgenommener Privatsphäre und Anonymität auf Forennutzer

Simone Braun, Andreas Schmidt, Valentin Zacharias

People Tagging - Aspekte und Möglichkeiten zur Gestaltung

\section{Perspektiven der MCI}

Peter Brödner, Markus Rohde, Gunnar Stevens, Volker Wulf

Perspektivwechsel auf IS: Von der Systemgestaltung zur Strukturation sozialer Praxis

Joel E. Fischer

Interrupting the Here and Now: Implications and Opportunities

Rüdiger Heimgärtner

Auf dem Weg zu einem_Erklärungsmodell kulturabhängiger Mensch-Maschine

Interaktion

\section{Anreize \& Veränderungspotenziale}

Stefan Seifert, Jan Krämer, Athanasios Mazarakis

Anreize zur Nutzung von Wikis in der Hochschullehre: Ergebnisse eines Pilotprojekts .....179

Matthias Laschke, Sarah Diefenbach, Stephanie Heidecker, Marc Hassenzahl

Transformationale Produkte: Acht Konzepte zum schonenden Umgang mit Ressourcen ...189

Stephanie Heidecker, Sarah Diefenbach, Philip Creutz, Matthias Laschke, Marc Hassenzahl

Transformationale Produkte: Erleben und wahrgenommene Veränderungspotentiale

\section{Modellbasierte Entwicklung und Adaption}

Anke Dittmar, Peter Forbrig

Repräsentation von Arbeit im modellgetriebenen versus -geleiteten Design .205

Marcel Dausend, Mark Poguntke

Spezifikation multimodaler Interaktionsanwendungen mit UML 
Dirk Veiel, Jörg M، Haake, Stephan Lukosch

Kontext-basierte Adaption von gemeinsamen Arbeitsbereichen. .225

\section{Einfache Interaktion für Alle}

Raphael Gfeller, Pascal Hauser

Rotated Lines: A Heatmap representation method for people affected by any kind of color blindness

Alexander Mertens, Bernhard Kausch, Nicole Jochems, Christopher Schlick

Selection Technique for Small Objects on Touch Screens for People with Muscle

Tremor.

Daniel Ullrich, Sarah Diefenbach

INTUI. Exploring the Facets of Intuitive Interaction.

\section{Usability-Evaluation}

Julia Jürgens, Thomas Mandl, Christa Womser-Hacker

Das Potenzial von Web Analytics für Usability-Evaluierungen.

Martin Schmettow, Cédric Bach, Dominique Scapin

Effizientere Usability Evaluationen mit gemischten Prozessen

\section{Mobil}

Knut Polkehn, Hartmut Wandke, Marcus Dahm

Usability-Evaluation interaktiver Geräte: Online vs. Labor?

Martin Pielot, Benjamin Poppinga, Björn Vester, Anastasia Kazakova, Lennard Brammer, Susanne Boll

Natch: A Watch-like Display for Less Distracting Pedestrian Navigation.

Julia Niemann, Jessika Reissland, Anja Neumann

Mobile Dienste im Fahrzeug: Gestaltung von Sprachausgaben zur Reduzierung visueller

Ablenkung.

\section{Be-Greifen}

Mathias Heilig, Mischa Demarmels, Katrin Allmendinger, Jens Gerken, Harald Reiterer

Fördern realitätsbasierte UIs kollaborative Rechercheaktivitäten?

Jens Heydekorn, Mathias Frisch, Raimund Dachselt

Eine Architektur zum flexiblen Einsatz von gestischer Interaktion

Philipp Heim, Thomas Schlegel, Thomas Ertl

Starke Kopplung: Interaktion als Schlüssel für das Semantic Web. 


\section{Methoden der MCI}

Steffi Beckhaus, Senana Lucia Brugger, Katharina Wolter

Die Artefaktkarte. 341

Martin Schrepp, Theo Held

Anwendung von GOMS-Analysen und CogTool in der Design-Praxis

Gregor Buchholz, Peter Forbrig

Analyse von Handlungsprotokollen zur Modellbildung.

\section{Systemdemonstrationen}

Matthias Rath, Ina Wechsung, Anja Naumann

Das SlidingWheel: Eine neue Interaktionsmetapher zur Navigation in großen

Datenmengen

Tanja Döring, Axel Sylvester, Albrecht Schmidt

Das „Soap Bubble Interface" - eine ephemere Benutzungsschnittstelle

Rainer Blum, Birgit Bomsdorf, Karim Khakzar, Dominik Rupprecht

Virtuelle Anprobe im Internet

\section{Workshops}

Jürgen Steimle, Johann Habakuk Israel, Bernard Robben

Be-greifbare Interaktionen in gemischten Wirklichkeiten.

David Elsweiler, Heiko Haller, Martin C. Kindsmüller, Richard Pircher

PKM 2010 - Workshop on_Personal Knowledge Management

Michael Herczeg

Workshop Basismodule und Basiscurricula für die Medieninformatik

Daniel Münter, Kyrill Meyer

Workshop „Human-Service-Interaction“

Alexander Nolte, Kai-Uwe Loser, Michael Koch, Nils Jeners, Florian Klompmaker

Nutzung interaktiver Displays im Kontext kollaborativer Arbeit

Alexander Richter, Angelika C. Bullinger, Michael Koch, Alexander Stocker

Soziotechnische Integration? Bottom Up? Simplicity? Was sind die Erfolgstreiber von

Enterprise 2.0?.

Jörg M. Haake, Tim Hussein, Volkmar Pipek, Stephan Lukosch

Workshop „Evaluation Adaptiver Systeme (EASYS)“

Autoren 